\title{
Can clinical features, ultrasound and elastography be useful for presurgical prediction of ovarian granulosa cell tumor: case report
}

\author{
Nabil Abdalla, Robert Piorkowski, Seweryn Trojanowski, Alicja Jakimiuk, Pawel Stanirowski, \\ Krzysztof Cendrowski, Wlodzimierz Sawicki \\ Chair and Clinic of Obstetrics, Gynecology and Oncology, II Faculty of Medicine, Medical \\ University of Warsaw, Poland.
}

A 39-year-old patient was admitted to our Oncology Centre for surgery because of right adnexal tumor. The patient suffered from heavy irregular menstrual cycles every 14-30 days, lasting 3 days for the last six months. The past medical, past surgical and family history was uneventful. The body mass index was 32. Clinical examination reveal movable right adnexal tumor of $7 \mathrm{~cm}$ diameter. The left adnexa and uterus were of normal size. The serum level of CA125 and HE4 was within normal range and achieved $5,3 \mathrm{IU} / \mathrm{ml}$ and $66,8 \mathrm{pmol} / \mathrm{L}$, respectively. Risk of malignancy algorithm (ROMA) revealed low risk $(13,1 \%)$ of ovarian malignancy. The level of serum estradiol, beta hCG, AFP and LDH was within normal range and reached $10 \mathrm{pg} / \mathrm{ml}, 1,2 \mathrm{mlU} / \mathrm{ml}, 2,85 \mathrm{ng} / \mathrm{ml}$ and $177 \mathrm{U} / \mathrm{L}$, respectively. The 2D ultrasound showed regular solid right adnexal tumor with dimensions of $71 \times 42 \times 75 \mathrm{~mm}$. The vascularization was assessed as grade 3 according to terms and definitions of International Ovarian Tumor Analysis (IOTA) group. Ultrasound with elastography option was used to assess the mass. The strain ratio of the anterior and posterior wall of the myometrium of the uterus compared to the adnexal mass was $0,17 \%$ and $0.17 \%$, respectively. There was no acoustic shadows, free fluid or evidence of metastases. The IOTA-ADNEX model predicted $32 \%$ risk of malignancy, with a probability of being borderline tumor, stage I ovarian cancer, stage II-IV ovarian cancer and metastases was $5,3 \%, 15,2 \%, 2,4 \%$ and $9,1 \%$, respectively. The mass was inconclusive according to the IOTA simple rules and subjective assessment of the expert ultrasonographist suggested malignant nature of the mass. After obtaining patient's consent, the patient underwent laparotomy, which confirmed soft yellow regular right ovarian solid mass without evidence of metastases. Right oviduct was without macroscopic changes. Complete right adnexal excision was performed and intraoperative histological assessment revealed mature type of ovarian granulosa cell tumor. Final histological examination confirmed the diagnosis of completely excised granulosa cell tumor without interruption of the tumor's capsule. Immunohistochemical staining showed positive staining for calretinin $(++)$, inhibin $(+)$ and smooth muscle actin $(++)$ and negative for CD117. The postoperative period was uneventful. Presurgical difficulties are attributed to contradicting finding from suspicious ultrasound appearance and normal level of tumor markers.
Elastography gave additional information about the tumor and it's consistency and compared it to the elastography features of the myometrium. Abnormal sudden change of menstrual cycle rhythm and heavy menstrual bleeding in a young patient with solid ovarian tumor suggested rather hormone secreting tumor. The final histological assessment confirmed rare adult type of ovarian granulosa cell tumor.

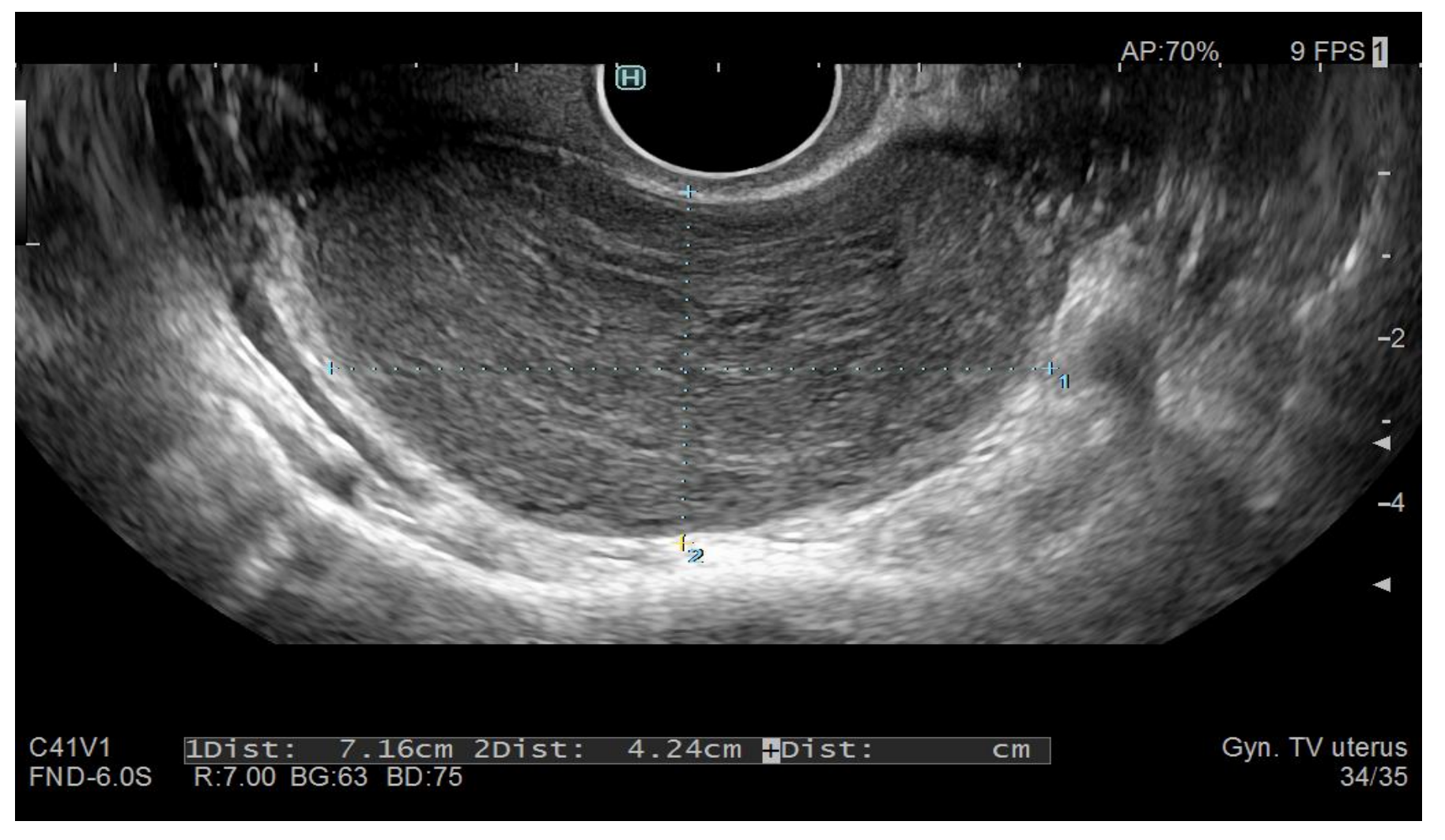

Regular solid right adnexal

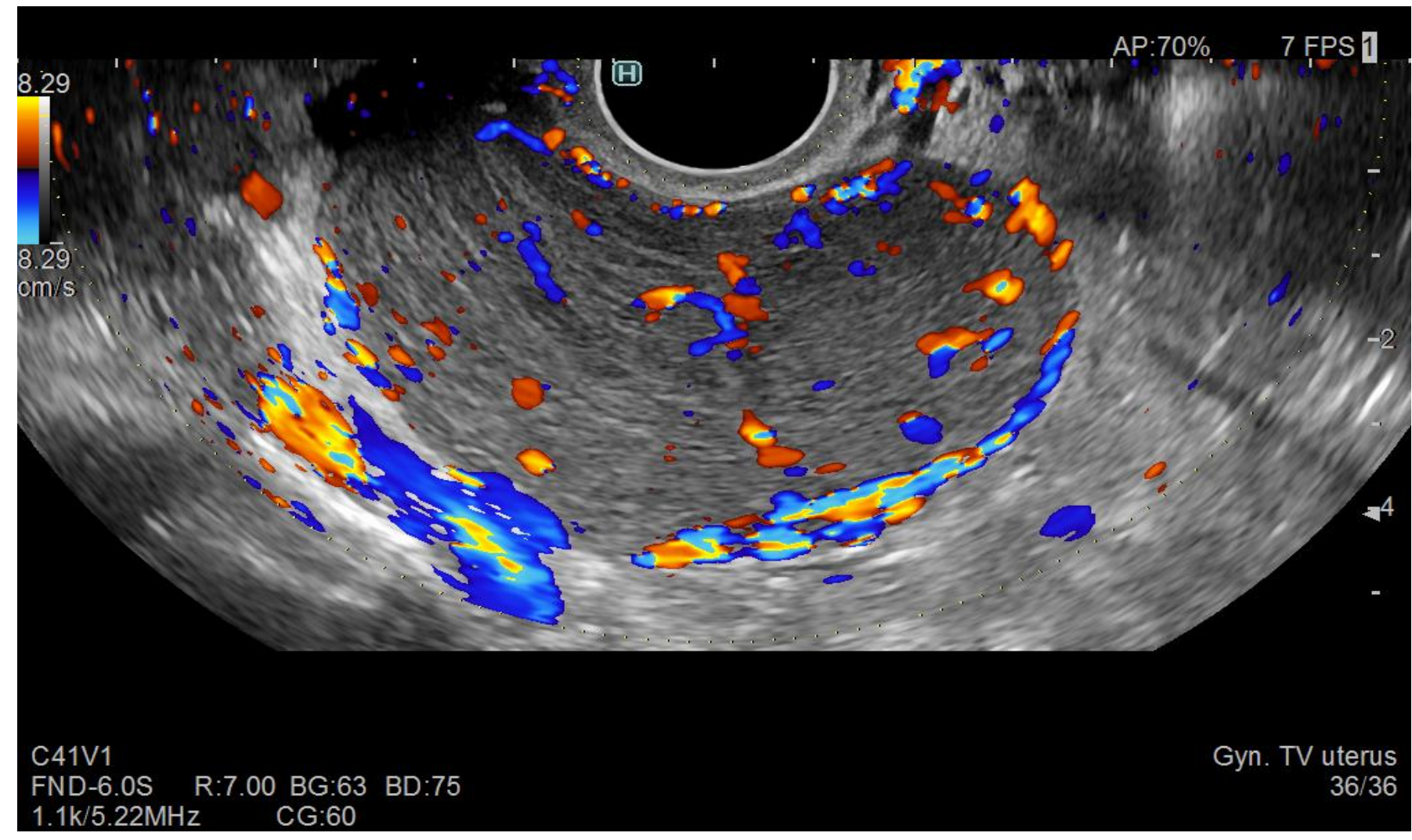

Vascularisation of the adnexal mass

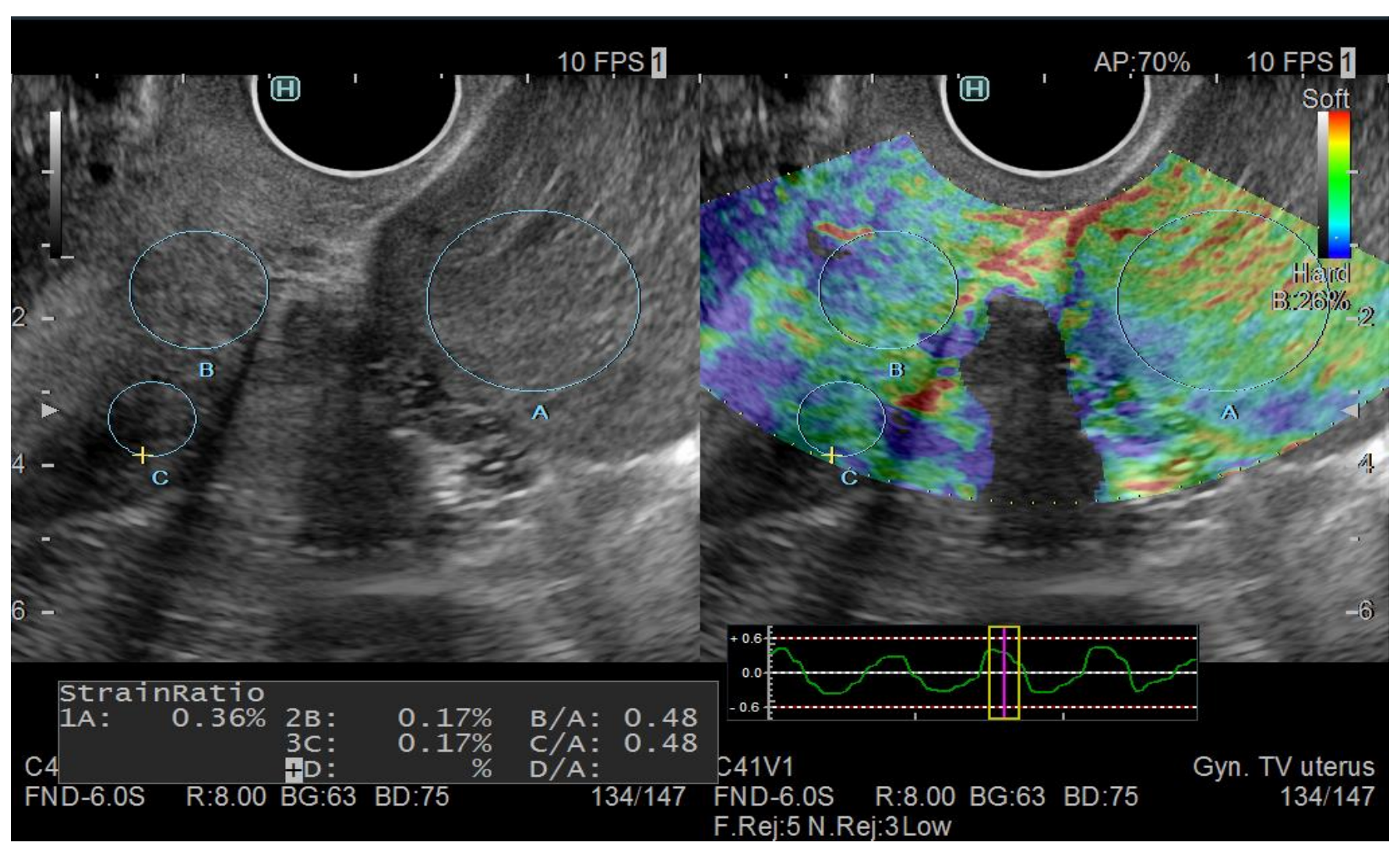

Elastography examination 